\title{
The Spleen: The Forgotten Organ in Acute Kidney Injury of Critical IIIness
}

\author{
Joseph C. Gigliotti Mark D. Okusa \\ Division of Nephrology, Center for Immunity, Inflammation and Regenerative Medicine, University of \\ Virginia Health System, Charlottesville, Va., USA
}

\section{Key Words \\ Cholinergic anti-inflammatory pathway - Acetylcholine . \\ Ultrasound · Inflammation · Sepsis}

\begin{abstract}
Acute kidney injury (AKI) is an increasing medical burden and is independently associated with mortality. AKI is a common comorbidity in the intensive care unit (ICU), with sepsisassociated AKI seen in almost a quarter of all ICU patients. Due to the high mortality seen in these patients, improved therapeutic options are needed. Data from experimental studies in animals support observations in humans that the host immune response to sepsis and trauma contributes to multiorgan failure and the high morbidity and mortality seen in critically ill patients. The spleen, a major component of the reticuloendothelial system, appears to be a key player in the 'cytokine storm' that develops after infection and trau$\mathrm{ma}$, and the resultant systemic inflammation is regulated by the autonomic nervous system. Over the past decade, evidence has suggested that controlling the splenic cytokine response improves tissue function and mortality in sepsis and other inflammatory-mediated diseases. One pathway that controls the response of the spleen to sepsis and trauma is the cholinergic anti-inflammatory pathway, and it may
\end{abstract}

provide a key target for therapeutic intervention. Here, we review this concept and highlight the potential use of ultrasound to stimulate the cholinergic anti-inflammatory pathway and reduce systemic inflammation and disease severity.

(c) 2014 S. Karger AG, Basel

\section{Acute Kidney Injury in Critically III Patients}

Acute kidney injury (AKI) remains a major public health burden and the rate of AKI continues to increase unabated [1]. AKI is a heterogeneous disorder and may be a consequence of hypotension, sepsis, trauma, medications, or nephrotoxins. Recent data from the National Hospital Discharge Survey of the National Center for Health Statistics on the number of hospitalized patients with a diagnosis of AKI (ICD-9 = 584.0 through 584.9) has increased over the last 20 years from 161,000 in 1990 to 2.7 million in 2010 , which is approximately $7 \%$ of hospitalized patients. In the intensive care unit (ICU), depending on the definition, AKI can occur in $1-25 \%$ of

Changing Paradigms in Acute Kidney Injury: From Mechanisms to Management - Proceedings of the 5th Annual UAB-UCSD O'Brien Center Symposium (San Diego, Calif., USA, March 4, 2014).

\section{KARGER}

E-Mail karger@karger.com www.karger.com/nec
(C) 2014 S. Karger AG, Basel

$1660-2110 / 14 / 1274-0153 \$ 39.50 / 0$
Mark D. Okusa, MD, FASN

PO Box 800133

Charlottesville, VA 22908-0133 (USA)

E-Mailmdo7y@virginia.edu 
patients and is associated with mortality rates of $15-60 \%$ $[2,3]$. Even those that survive are at a higher risk for dialysis or death [4]. Sepsis-associated AKI is high in ICU patients and occurs in 15-20\% of all ICU admissions, and the mortality rate of these critically ill patients is approximately $70 \%$ compared to $45 \%$ in patients with AKI alone [5]. The high mortality rate of these individuals is thought to be due to multiorgan dysfunction. Here, the spleen appears to be a critical organ at the crossroad between bacterial infection and multi-organ dysfunction. This review will focus on the important role of the spleen in sepsis and AKI.

\section{Splenic Function in Sepsis}

The spleen is a major component of the reticuloendothelial system. Once bacteria/bacterial products have penetrated the initial barriers (epithelium and tissue resident phagocytes) and gained access to the blood, the next line of defense is the reticuloendothelial system. The reticuloendothelial system refers to tissues containing phagocytic myeloid cells (presumably macrophages), which play a key role in 'filtering the blood', and primarily include the spleen, liver, lung, and peritoneum. While the liver is by far the largest of these organs, with the greatest capacity for phagocytosis, the red pulp of the spleen has been shown to be more efficient at removing debris and particulate matter in the blood on a per-gram basis $[6,7]$. The white pulp contains several structures involved in antibody production, antigen presentation, and $\mathrm{T}$ cell activation.

Upon bacterial sequestration by phagocytes, tissue expression of proinflammatory cytokines ensues [interleukin (IL)-1, IL-6, IL-8, tumor necrosis factor (TNF)- $\alpha$, and IL-12] and elevated plasma levels are detected within hours $[8,9]$. Given the importance of the reticuloendothelial system in bacterial engulfment, large amounts of cytokine are produced in these tissues, with peak TNF- $\alpha$ mRNA expression occurring around $3 \mathrm{~h}$ after septic surgery or lipopolysaccharide injection in mice [10]. At the protein level, tissue TNF- $\alpha$ expression is easily detected within $90 \mathrm{~min}$, with the spleen being reported to produce approximately 10 -fold more TNF- $\alpha$ than the liver and lung on a per-gram-of-tissue basis [11]. Several animal studies have demonstrated that deficiencies in inflammatory components improve survival in sepsis/endotoxemia $[12,13]$. Because of a number of failed clinical trials in sepsis, a better understanding of the different immunological phases of sepsis is needed and new strategies need to be considered [14]. Furthermore, more studies need be conducted regarding the pathogenesis of AKI and in particular septic AKI.

An ongoing debate challenges our concept of the inflammatory response to sepsis and trauma [14]. Although early on the focus was on the infectious agent, Thomas [15] was responsible for a paradigm shift by changing the focus from pathogens to the pathological dysregulated host response that serves as the basis for the clinical expression of the systemic inflammatory response syndrome (SIRS). 'When we sense lipopolysaccharide, we are likely to turn on every defense at our disposal, we will bomb, defoliate, blockade, seal off and destroy all the tissues in the area' [15]. The 'defense system' is set into motion in response to danger-associated molecular patterns or pathogen-associated molecular patterns that activate innate immune cells to produce a variety of pro- and anti-inflammatory cytokines and prime the ensuing adaptive immune response [15]. Currently, two competing views prevail that describe the host inflammatory response to sepsis and trauma. The first view is that there is an immediate activation of both pro- and anti-inflammatory immune responses at the onset of sepsis through activation of the innate immunity. Early deaths are due typically to a hyperinflammatory 'cytokine storm' with clinical features such as fever, circulatory collapse, acidosis, and hypercatabolism. If sepsis persists, a pronounced immunosuppression ensues due to failure of the innate and adaptive immunity leading to uncontrolled infection and death. The second view is that early and persistent activation of the innate immunity and suppression of the adaptive immunity are thought to occur after the onset of sepsis. Death is due to the persistent activation of innate immunity leading to uncontrolled inflammation and tissue and organ injury $[14,16]$.

\section{The Spleen in AKI}

In AKI, the role of the spleen has been demonstrated in experimental studies in splenectomized animals. Faubel and coworkers demonstrated that splenectomy did not exacerbate AKI; however, there was an increase in lung permeability [17]. In addition, they found that following ischemia-reperfusion injury (IRI) the splenic IL-10 production was increased, suggesting that the spleen contributes to the anti-inflammatory response and the reduction of lung injury [17]. In preliminary studies, we demonstrated that anesthetized animals that underwent splenectomy 1 week prior to mild bilateral renal IRI suffered 
Fig. 1. Proposed mechanism of ultrasoundinduced protection of IRI. Activation of the adrenergic splenic nerve results in the release of norepinephrine, which binds to adrenergic receptors on nearby $\mathrm{CD} 4+\mathrm{T}$ cells. This stimulates the production of acetylcholine that binds $\alpha 7 \mathrm{nAChRs}$ on myeloid cells (macrophages) and results in reduced inflammation and IRI. NE $=$ Norepinephrine; $\mathrm{ACh}=$ acetylcholine; $\mathrm{BT}=\alpha$-bungarotoxin; Adr $\mathrm{R}=$ adrenergic receptor. Adapted from Gigliotti et al. [28].

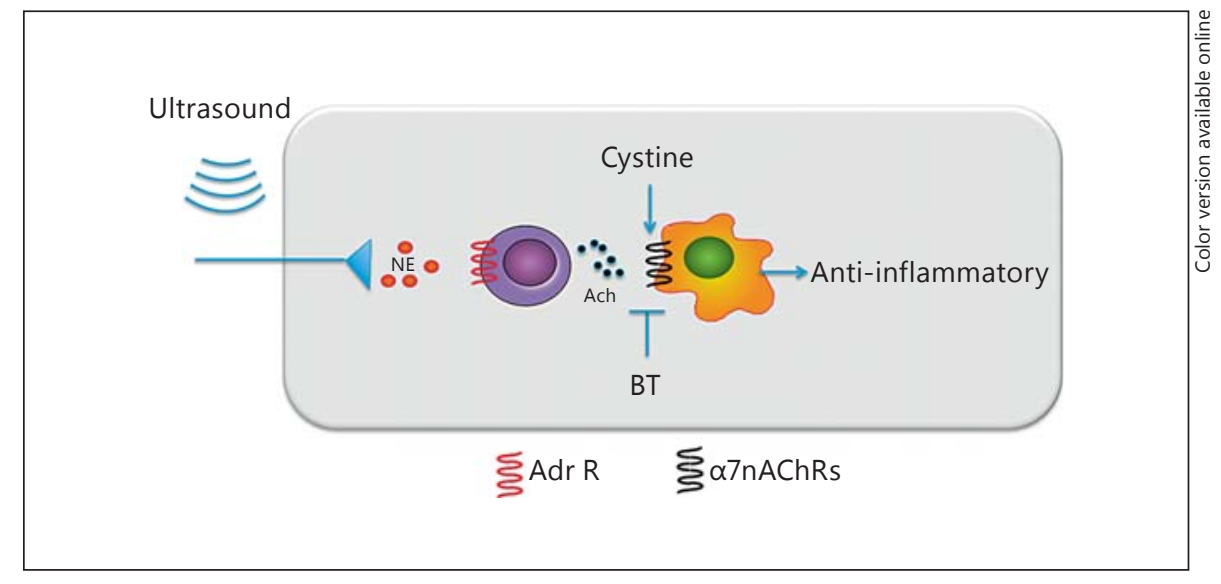

worse AKI, higher circulating and renal infiltrating neutrophils, and increased kidney expression of CXCL1 and IL-6. These results support the concept that the spleen plays an important role not only in sepsis-associated AKI but also in AKI from IRI [Gigliotti et al., unpubl. observations]. Not all studies, however, have shown a protective role of the spleen in organ injury. Studies in hepatic injury show that splenectomy protects the liver from injury [18].

\section{Neural Control of Systemic Inflammation via the Spleen}

Prior work by Straub [19] and more recently by Andersson and Tracey [20] has shed light on an important interaction between the nervous and immune systems. The nervous system, composed of both afferent sensory and efferent effector mechanisms, controls a number of functions such as voluntary movements, visceral function, and the heart rate to maintain normal homeostasis. In a similar manner, the nervous system controls inflammatory responses through a reflex circuit in which afferent signals sense inflammation and efferent signals quell inflammation. Immune cells express a variety of neurotransmitter receptors [21-24] which are modulated based on the activation status. In general, it appears that electrical stimulation of splenic tissue results in a reduced cytokine production when challenged with inflammatory stimuli. This has been shown in ex vivo tissue stimulation [19] as well as in in vivo electrical stimulation via the vagus nerve [25]. The mechanism for this immunosuppression has since been described and the efferent signal is referred to as the cholinergic anti-inflammatory pathway [26]. Afferent and efferent signals are transmitted by the vagus nerve in response to danger-associated molecular patterns or pathogen-associated molecular patterns. Activation of the splenic nerve stimulates the production of acetylcholine by a splenic CD4+ subset within the white pulp via $\beta$-adrenergic receptor activation. Acetylcholine then crosses the marginal zone into the red pulp and activates $\alpha-7$ nicotinic acetylcholine receptors ( $\alpha 7 \mathrm{nAChRs)}$ on splenic macrophages. Binding of acetylcholine to a $7 \mathrm{nAChRs}$ on nearby macrophages results in suppressed splenic and in turn systemic cytokine (proinflammatory cytokines such as TNF- $\alpha$, IL- $1 \beta$, and high-mobility group box 1) levels during inflammation (both sepsis and other inflammatory diseases). Experimental studies have demonstrated that stimulation of the vagus nerve attenuates cytokine release in sepsis, IRI, and other states of inflammation.

\section{Alternative Methods to Stimulate the Cholinergic Anti-Inflammatory Pathway}

While this pathway seems promising, the current methods used to directly stimulate the cholinergic anti-inflammatory pathway seem limited to administration of cholinergic agonists or surgical methods to stimulate the vagus nerve [27]. Recently, we observed that mice exposed to a modified ultrasound regimen were protected from IRI [28]. The treatment was effective and prevented both acute tissue injury and the ensuing fibrotic response. We exposed anesthetized mice to an ultrasound protocol $24 \mathrm{~h}$ before renal IRI. Ultrasound-treated mice exhibited preserved kidney morphology and function compared to sham-treated mice. Components of the cholinergic antiinflammatory pathway appeared to mediate the tissue- 
protective effect of the ultrasound treatment. Splenectomy and adoptive transfer studies revealed that the spleen and $\mathrm{CD} 4+\mathrm{T}$ cells mediated the protective effects of ultrasound. Blockade or genetic deficiency of the $a 7$ nicotinic acetylcholine receptor abrogated the protective effect of ultrasound and an $\alpha 7$ nicotinic acetylcholine receptor agonist mimicked the effect of ultrasound. These results suggest that an ultrasound-based treatment could have therapeutic potential for the prevention of AKI, possibly by stimulating the splenic cholinergic anti-inflammatory pathway. The advantage of this method is that it is a nonpharmacologic, noninvasive, ultrasound-based approach that is simple, inexpensive and portable. Whether ultrasound can attenuate sepsis-associated AKI has not yet been determined.

\section{Conclusion}

AKI is a growing medical concern, especially among individuals in the ICU. Inflammation appears to play a key role in the pathogenesis of AKI, and improved methods of immune-modulation are needed. The spleen appears to play an important role in cytokine production and disease progression, suggesting that it is a potential therapeutic target for a range of diseases. Recent evidence suggests that the cholinergic anti-inflammatory pathway is an inherent anti-inflammatory function of the spleen and is capable of modulating systemic inflammation via pharmacological or electrical stimulation. Our recent data suggests that ultrasound is also capable of stimulating this pathway and reduces systemic inflammation in an animal model of AKI. Thus, targeting the splenic cholinergic anti-inflammatory pathway using ultrasoundbased technologies is a promising avenue for future therapeutic development. The therapeutic potential extends beyond the kidney, and additional research targeting inflammatory disorders such as myocardial infarction, acute lung injury, rheumatoid arthritis, inflammatory bowel disease, and other disorders is needed.

\section{Acknowledgment}

We would like to thank Dr. Diane L. Rosin (Department of Pharmacology), Dr. John Hossack (Department of Biomedical Engineering), Dr. Sasha Klibanov (Department of Medicine), and Dr. Kambiz Kalantari (Department of Medicine) for their expert advice. This work was supported by National Institutes of Health grants R01 DK062324, R21 093841, and T32 DK072922.

\section{References}

1 Hsu RK, McCulloch CE, Dudley RA, Lo LJ, Hsu CY: Temporal changes in incidence of dialysis-requiring AKI. J Am Soc Nephrol 2013;24:37-42.

2 Singbartl K, Kellum JA: Aki in the ICU: definition, epidemiology, risk stratification, and outcomes. Kidney Int 2012;81:819-825.

- 3 Cerda J, Lameire N, Eggers P, Pannu N, Uchino S, Wang H, Bagga A, Levin A: Epidemiology of acute kidney injury. Clin J Am Soc Nephrol 2008;3:881-886.

-4 Coca SG, Yusuf B, Shlipak MG, Garg AX, Parikh CR: Long-term risk of mortality and other adverse outcomes after acute kidney injury: a systematic review and meta-analysis. Am J Kidney Dis 2009;53:961-973.

5 Uchino S, Kellum JA, Bellomo R, Doig GS, Morimatsu H, Morgera S, Schetz M, Tan I, Bouman C, Macedo E, Gibney N, Tolwani A, Ronco C: Acute renal failure in critically ill patients: a multinational, multicenter study. JAMA 2005;294:813-818.

6 Thompson PN, Cho E, Blumenstock FA, Shah DM, Saba TM: Liver and spleen phagocytic depression after peripheral ischemia and reperfusion. Am J Surg 1992;164:248253.
7 Litzinger DC, Buiting AM, van Rooijen N, Huang L: Effect of liposome size on the circulation time and intraorgan distribution of amphipathic poly(ethylene glycol)-containing liposomes. Biochim Biophys Acta 1994; 1190:99-107.

-8 Hegde A, Uttamchandani M, Moochhala SM, Bhatia M: Plasma cytokine profiles in preprotachykinin-A knockout mice subjected to polymicrobial sepsis. Mol Med 2010;16:4552.

-9 Mukherjee R, McQuinn TC, Dugan MA, Saul JP, Spinale FG: Cardiac function and circulating cytokines after endotoxin exposure in neonatal mice. Pediatr Res 2010;68:381-386.

10 Hadjiminas DJ, McMasters KM, Peyton JC, Cheadle WG: Tissue tumor necrosis factor mRNA expression following cecal ligation and puncture or intraperitoneal injection of endotoxin. J Surg Res 1994;56:549-555.

-11 Huston JM, Ochani M, Rosas-Ballina M, Liao H, Ochani K, Pavlov VA, Gallowitsch-Puerta M, Ashok M, Czura CJ, Foxwell B, Tracey KJ, Ulloa L: Splenectomy inactivates the cholinergic antiinflammatory pathway during lethal endotoxemia and polymicrobial sepsis. J Exp Med 2006;203:1623-1628.
2 Ulloa L: The vagus nerve and the nicotinic anti-inflammatory pathway. Nat Rev Drug Discov 2005;4:673-684.

13 Hotchkiss RS, Karl IE: The pathophysiology and treatment of sepsis. N Engl J Med 2003; 348:138-150.

14 Hotchkiss R, Monneret G, Payen D: Sepsisinduced immunosuppression: from cellular dysfunctions to immunotherapy. Nat Rev Immunol 2013;13:862-874.

15 Thomas L: Germs. N Engl J Med 1972;287: 553-555.

-16 Xiao W, Mindrinos M, Seok J, Cuschieri J, Cuenca A, Gao H, Hayden D, Hennessy L, Moore E, Minei J, Bankey P, Johnson J, Sperry J, Nathens A, Billiar T, West M, Brownstein B, Mason P, Baker H, Finnerty C, Jeschke M, López M, Klein M, Gamelli R, Gibran N, Arnoldo B, Xu W, Zhang Y, Calvano S, McDonald-Smith G, Schoenfeld D, Storey J, Cobb J, Warren H, Moldawer L, Herndon D, Lowry S, Maier R, Davis R, Tompkins RG; Inflammation and Host Response to Injury Large-Scale Collaborative Research Program: A genomic storm in critically injured humans. J Exp Med 2011;208:2581-2590. 
17 Andres-Hernando A, Altmann C, Ahuja N, Lanaspa MA, Nemenoff R, He Z, Ishimoto T, Simpson PA, Weiser-Evans MC, Bacalja J, Faubel S: Splenectomy exacerbates lung injury after ischemic acute kidney injury in mice. Am J Physiol Renal Physiol 2011; 301:F907-F916.

18 Jiang H, Meng F, Li W, Tong L, Qiao H, Sun $\mathrm{X}$ : Splenectomy ameliorates acute multiple organ damage induced by liver warm ischemia reperfusion in rats. Surgery 2007;141:3240.

19 Straub RH: Complexity of the bi-directional neuroimmune junction in the spleen. Trends Pharmacol Sci 2004;25:640-646.

20 Andersson U, Tracey KJ: Reflex principles of immunological homeostasis. Annu Rev Immunol 2012;30:313-335.
1 Kin NW, Sanders VM: It takes nerve to tell T and B cells what to do. J Leukoc Biol 2006;79: 1093-1104.

22 Maestroni GJ: Sympathetic nervous system influence on the innate immune response. Ann NY Acad Sci 2006;1069:195-207.

23 Kawashima K, Yoshikawa K, Fujii YX, Moriwaki Y, Misawa H: Expression and function of genes encoding cholinergic components in murine immune cells. Life Sci 2007;80:23142319.

24 Koval LM, Zverkova AS, Grailhe R, Utkin YN, Tsetlin VI, Komisarenko SV, Skok MV: Nicotinic acetylcholine receptors alpha4beta2 and alpha7 regulate myelo- and erythropoiesis within the bone marrow. Int J Biochem Cell Biol 2008;40:980-990.

25 Tracey KJ: Physiology and immunology of the cholinergic antiinflammatory pathway. J Clin Invest 2007;117:289-296.
26 Rosas-Ballina M, Olofsson PS, Ochani M, Valdes-Ferrer SI, Levine YA, Reardon C, Tusche MW, Pavlov VA, Andersson U, Chavan S, Mak TW, Tracey KJ: Acetylcholinesynthesizing $\mathrm{T}$ cells relay neural signals in a vagus nerve circuit. Science 2011;334:98-101.

27 Bonaz B, Picq C, Sinniger V, Mayol JF, Clarençon D: Vagus nerve stimulation: from epilepsy to the cholinergic anti-inflammatory pathway. Neurogastroenterol Motil 2013;25: 208-221.

28 Gigliotti JC, Huang L, Ye H, Bajwa A, Chattrabhuti K, Lee S, Klibanov AL, Kalantari K, Rosin DL, Okusa MD: Ultrasound prevents renal ischemia-reperfusion injury by stimulating the splenic cholinergic anti-inflammatory pathway. J Am Soc Nephrol 2013;24: 1451-1460. 